\title{
A unified theory for surface emitting chirped circular grating lasers
}

\author{
Xiankai Sun* and Amnon Yariv \\ Department of Applied Physics, California Institute of Technology, Pasadena, CA, USA 91125
}

\begin{abstract}
We develop a unified theory to analyze the modal properties of surface emitting chirped circular grating lasers. Based on solving the resonance conditions which involve two types of reflectivities of chirped circular gratings, this theory is both easy to understand and convenient to apply to different configurations of circular grating lasers. Though in a more concise format, this approach is shown to be in agreement with previous derivations which use the characteristic equations. With this unified analysis, the modal properties of circular DFB, disk-, and ring- Bragg resonator lasers are obtained and compared.
\end{abstract}

Keywords: Surface emitting lasers, unified theory, laser modes, grating reflectivity, Bragg resonators, semiconductor lasers, integrated optics.

\section{INTRODUCTION}

Surface emitting circular grating lasers with large emission apertures have attracted considerable attention because of their capabilities in producing circularly-symmetric, narrow-divergence laser beams. In such lasers, the grating serves two purposes: providing feedback for the in-plane fields to form a radial resonator and secondly acting as a broadside output coupler. Due to the inherent high values of $Q^{\prime V}(Q$, quality factor of resonator mode; $V$, modal volume), these lasers are excellent candidates for low-threshold, single-mode operation taking advantage of the large Purcell effect [1].

In conventional circular resonators, $Q$ and $V$ are related due to the "bending loss" problem where smaller radii lead to increased radiation losses [2]. In 2003, we proposed that the optimal confinement of the optical fields in the radial direction be achieved by radially chirped gratings $[3,4]$. Devices implementing this design have demonstrated both a low threshold and a small modal volume [5,6]. A comprehensive coupled-mode theory, including the effects of vertical radiation, of such circular grating lasers has been derived and used for analyzing disk and ring types Bragg resonator lasers $[7,8]$. The characteristic equations used for obtaining the threshold gains and detuning factors were derived by matching the electric fields and their first derivatives at all the interfaces between grating and no-grating regions. However, the complexity of the characteristic equations rendered them difficult to analyze and interpret intuitively. Moreover, though the circular DFB, disk-, and ring- Bragg resonator lasers all belong to the family of circular grating lasers, they were not comparable because the analyses used to treat them were not directly related.

In this paper, we aim at developing a unified theory which can simplify the analysis of modal properties of different configurations of the surface emitting chirped circular grating lasers and establish the relations between them. In Sec. 2, a brief review of derivations of the characteristic equations for the circular grating lasers serves a background and those equations will be used as the benchmarks for the new theory. In Sec. 3, the coupled-wave equations are solved under two typical circular boundary conditions obtaining two types of reflectivities for the chirped circular Bragg gratings, which are then employed to derive the resonance conditions. The resonance condition theory is shown to be in agreement with the characteristic equations. Lastly the resonant modes of circular DFB, disk-, and ring- Bragg resonator lasers are numerically obtained, and their threshold gains and frequency detuning factors are compared and discussed.

\section{BRIEF REVIEW OF DERIVATIONS OF CHARACTERISTIC EQUATIONS FOR CHIRPED CIRCULAR GRATING LASERS}

\footnotetext{
*xksun@caltech.edu; phone 1626 395-4414; fax 1 626 405-0928; http://www.its.caltech.edu/ xksun
} 


\subsection{Coupled-wave equations for chirped circular Bragg gratings}

The structures to be analyzed here are illustrated in Fig. 1. There are essentially three configurations of circular grating lasers: (a) Circular DFB lasers - the grating extends from the center to the exterior boundary $x_{b}$. (b) Disk Bragg resonator lasers - a center disk is surrounded by a radial Bragg grating extending from $x_{0}$ to $x_{b}$. (c) Ring Bragg resonator lasers - an annular defect is surrounded by inner and outer gratings on both sides. The inner grating extends from the center to $x_{L}$ while the outer from $x_{R}$ to $x_{b}$. In order to optimally interact with the optical fields, the gratings have to be designed to match the phase of the modal fields $[3,4]$. The eigenmodes of the wave equation in cylindrical coordinates, the Bessel functions, have nonperiodic zeros, yielding a radial chirp modulation of the refractive index, i.e., chirped gratings.
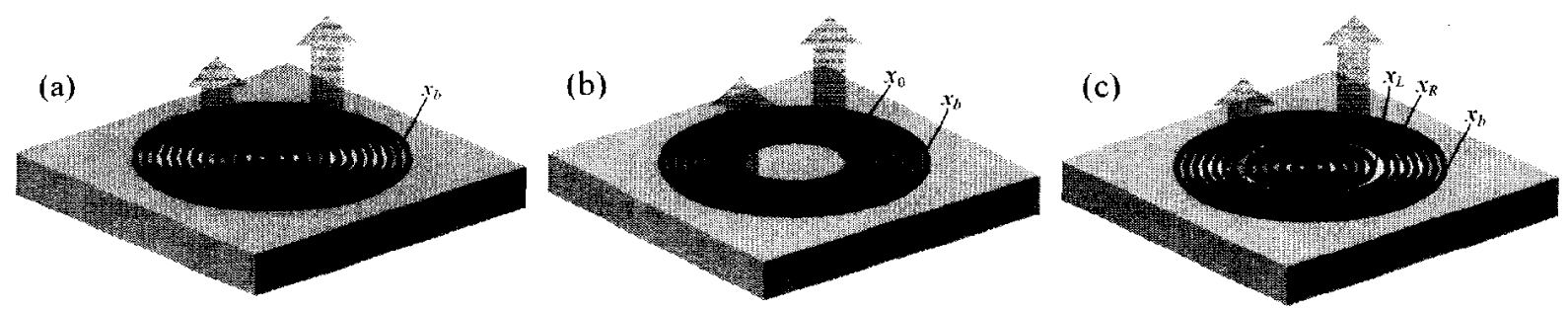

Fig. 1. Surface emitting chirped circular grating lasers: (a) Circular DFB laser; (b) Disk Bragg resonator laser; (c) Ring Bragg resonator laser. Laser radiation is coupled out of the resonators in vertical direction via the gratings.

Including the effect of resonant vertical radiation, we derived in [7] a comprehensive coupled-mode theory for such chirped circular grating structures in active media. The effect of vertical radiation is incorporated into the coupled inplane wave equations by a numerical Green's function method. The in-plane (vertically confined) electric field is expressed as $E(x)=A(x) H_{m}^{(1)}(x)+B(x) H_{m}^{(2)}(x) \cdot H_{m}^{(1)}(x)$ and $H_{m}^{(2)}(x)$ are the Hankel functions which represent the in-plane outward- and inward- propagating cylindrical waves. A set of evolution equations for the amplitudes $A(x)$ and $B(x)$ is obtained:

$$
\left\{\begin{array}{l}
\frac{d A(x)}{d x}=u A(x)-v B(x) e^{2 i \delta \cdot x} \\
\frac{d B(x)}{d x}=-u B(x)+v A(x) e^{-2 i \delta \cdot x} .
\end{array}\right.
$$

In the above equations, $x$ is the normalized radius $x=\beta \rho . \delta=\left(\beta_{\text {design }}-\beta\right) / \beta$ denotes the normalized frequency detuning factor, representing a relative frequency shift of the resonant mode from the optimal coupling design. The coefficients $u$ and $v$ are defined as $u=g_{A}-h_{1}, v=h_{1}+i h_{2}$, where $g_{A}$ is the normalized gain coefficient. The minimum value of $g_{A}$ required to achieve laser emission will be determined by the characteristic equations or resonance conditions. $h_{1}$ and $h_{2}$ are the radiation- and feedback- coupling coefficients, respectively. It should be noted that, although Eqs. (1) appear to be a set of coupled equations for in-plane waves only, they implicitly include the effect of vertical radiation due to $h_{1}$. When obtaining the thresholds for the laser modes, vertical radiation is treated as a loss term [7].

\subsection{Boundary conditions and characteristic equations for chirped circular grating lasers}

In grating regions Eqs. (1) can be analytically solved yielding the generic solutions for the amplitudes $A(x), B(x)$, and thus the expression for electric field $E(x)[7]$ :

$$
\left\{\begin{array}{l}
A(x)=\left[C_{1} e^{S x}+C_{2} e^{-S x}\right] e^{i \delta \cdot x} \\
B(x)=-\frac{1}{v}\left[C_{1}(S-u+i \delta) e^{S x}-C_{2}(S+u-i \delta) e^{-S x}\right] e^{-i \delta \cdot x},
\end{array}\right.
$$

where $S \equiv \sqrt{(u-i \delta)^{2}-v^{2}}$. 
In no-grating regions where $h_{1}=h_{2}=0,(1)$ is reduced to

whose solution is

$$
\left\{\begin{array}{l}
\frac{d A(x)}{d x}=g_{A} A(x) \\
\frac{d B(x)}{d x}=-g_{A} B(x)
\end{array}\right.
$$

where $A_{0}$ and $B_{0}$ are integration constants.

$$
\left\{\begin{array}{l}
A(x)=A_{0} e^{g_{4} x} \\
B(x)=B_{0} e^{-g_{4} x}
\end{array}\right.
$$

Next different boundary conditions will be applied for each configuration of circular grating lasers to obtain the characteristic equations.

1. Circular DFB lasers:

The in-plane electric field simply takes the form of $E(x)=A(x) H_{m}^{(1)}(x)+B(x) H_{m}^{(2)}(x)$ for $0<x<x_{b}$. The boundary conditions only require that the total field be finite at the center $x=0$ and the incoming wave vanish at the exterior boundary $x=x_{b}$. Therefore by enforcing $A(0)=B(0)$ and $B\left(x_{b}\right)=0$, the characteristic equation is

2. Disk Bragg resonator lasers:

$$
-\quad \frac{S-u+v+i \delta}{S+u-v-i \delta}=\frac{S-u+i \delta}{S+u-i \delta} e^{2 S \cdot x_{b}} .
$$

The in-plane electric field takes the form of

$$
E(x)=\left\{\begin{array}{lll}
A_{1} e^{g_{A} x} H_{m}^{(1)}(x)+B_{1} e^{-g_{A} x} H_{m}^{(2)}(x), & \text { for } 0<x<x_{0} \\
A_{2}(x) H_{m}^{(1)}(x)+B_{2}(x) H_{m}^{(2)}(x), & \text { for } x_{0}<x<x_{b}
\end{array}\right.
$$

Boundary conditions: in addition to those two required in the circular DFB lasers $\left(A_{1}=B_{1}\right.$ and $\left.B_{2}\left(x_{b}\right)=0\right)$, the electric field and its first derivative in this case have to be continuous at the interface $x_{0}$. This results in the characteristic equation for the modes of disk Bragg resonator lasers:

where

$$
\frac{e^{2 g_{A} x_{0}} H_{m}^{(1)}\left(x_{0}\right)+H_{m}^{(2)}\left(x_{0}\right)}{\left(g_{A}+i\right)\left[e^{2 g_{A} x_{0}} H_{m}^{(1)}\left(x_{0}\right)-H_{m}^{(2)}\left(x_{0}\right)\right]}=\frac{\xi}{\zeta}
$$

$$
\xi=\left[1+\frac{S-u+i \delta}{S+u-i \delta} e^{2 S\left(x_{h}-x_{0}\right)}\right] e^{2 i \delta \cdot x_{0}} H_{m}^{(1)}\left(x_{0}\right)-\frac{(S-u+i \delta)}{v}\left[1-e^{2 S\left(x_{h}-x_{0}\right)}\right] H_{m}^{(2)}\left(x_{0}\right)
$$

and

$$
\begin{array}{r}
\zeta=\left[S+i \delta-(S-i \delta) \frac{S-u+i \delta}{S+u-i \delta} e^{2 S\left(x_{b}-x_{0}\right)}\right] e^{2 i \delta \cdot x_{0}} H_{m}^{(1)}\left(x_{0}\right)+i\left[1+\frac{S-u+i \delta}{S+u-i \delta} e^{2 S\left(x_{b}-x_{0}\right)}\right] e^{2 i \delta \cdot x_{0}} H_{m}^{(1)}\left(x_{0}\right) \\
-\frac{(S-u+i \delta)}{v}\left[S-i \delta+(S+i \delta) e^{2 S\left(x_{b}-x_{0}\right)}\right] H_{m}^{(2)}\left(x_{0}\right)+i \frac{(S-u+i \delta)}{v}\left[1-e^{2 S\left(x_{b}-x_{0}\right)}\right] H_{m}^{(2)}\left(x_{0}\right) .
\end{array}
$$

\section{Ring Bragg resonator lasers:}

The in-plane electric field takes the form of

$$
E(x)= \begin{cases}A_{1}(x) H_{m}^{(1)}(x)+B_{1}(x) H_{m}^{(2)}(x), & \text { for } 0<x<x_{L} \\ A_{2} e^{g_{A} x} H_{m}^{(1)}(x)+B_{2} e^{-g_{A} x} H_{m}^{(2)}(x), & \text { for } x_{L}<x<x_{R} \\ A_{3}(x) H_{m}^{(1)}(x)+B_{3}(x) H_{m}^{(2)}(x), & \text { for } x_{R}<x<x_{b}\end{cases}
$$


Boundary conditions: in addition to those two required in the circular DFB lasers $\left(A_{1}(0)=B_{1}(0)\right.$ and $\left.B_{3}\left(x_{b}\right)=0\right)$, the electric field and its first derivative in this case have to be continuous at both interfaces $x_{L}$ and $x_{R}$. This results in the characteristic equation for the modes of ring Bragg resonator lasers:

where

$$
\frac{\left(g_{A}+i\right)(L H S)_{1}-1}{\left(g_{A}+i\right)(L H S)_{1}+1} \cdot \frac{\left(g_{A}+i\right)(R H S)_{I I}+1}{\left(g_{A}+i\right)(R H S)_{I I}-1}=\frac{H_{m}^{(1)}\left(x_{R}\right)}{H_{m}^{(2)}\left(x_{R}\right)} \cdot \frac{H_{m}^{(2)}\left(x_{L}\right)}{H_{m}^{(1)}\left(x_{L}\right)} e^{2 g_{A}\left(x_{R}-x_{L}\right)},
$$

$$
(L H S)_{I}=\frac{\left\{\begin{array}{c}
{\left[e^{S \cdot x_{L}+i \delta \cdot x_{L}}+\frac{S-u+v+i \delta}{S+u-v-i \delta} e^{-S \cdot x_{L}+i \delta \cdot x_{L}}\right] H_{m}^{(1)}\left(x_{L}\right)} \\
-\frac{1}{v}\left[(S-u+i \delta) e^{S \cdot x_{L}-i \delta \cdot x_{L}}-\frac{S-u+v+i \delta}{S+u-v-i \delta}(S+u-i \delta) e^{-S \cdot x_{i}-i \delta \cdot x_{L}}\right] H_{m}^{(2)}\left(x_{L}\right)
\end{array}\right\}}{\left\{\begin{array}{l}
{\left[(S+i(\delta+1)) e^{S \cdot x_{L}+i \delta \cdot x_{L}}+\frac{S-u+v+i \delta}{S+u-v-i \delta}(-S+i(\delta+1)) e^{-S \cdot x_{L}+i \delta \cdot x_{L}}\right] H_{m}^{(1)}\left(x_{L}\right)} \\
-\frac{1}{v}\left[(S-u+i \delta)(S-i(\delta+1)) e^{S \cdot x_{L}-i \delta \cdot x_{L}}+\frac{S-u+v+i \delta}{S+u-v-i \delta}(S+u-i \delta)(S+i(\delta+1)) e^{-S \cdot x_{L}-i \delta \cdot x_{L}}\right] H_{m}^{(2)}\left(x_{L}\right)
\end{array}\right\}}
$$

and

$$
(R H S)_{m}=\frac{e^{S \cdot x_{R}+i \delta \cdot x_{R}}\left[1+\frac{S-u+i \delta}{S+u-i \delta} e^{2 S\left(x_{b}-x_{R}\right)}\right] H_{m}^{(1)}\left(x_{R}\right)-\frac{(S-u+i \delta)}{v} e^{S \cdot x_{R}-i \delta \cdot x_{R}}\left[1-e^{2 S\left(x_{b}-x_{R}\right)}\right] H_{m}^{(2)}\left(x_{R}\right)}{\left\{\begin{array}{l}
(S+i(\delta+1)) e^{S \cdot x_{R}+i \delta \cdot x_{R}}\left[1+\frac{S-u+i \delta}{S+u-i \delta} e^{2 S\left(x_{b}-x_{R}\right)}\right] H_{m}^{(1)}\left(x_{R}\right)-2 S \frac{S-u+i \delta}{S+u-i \delta} e^{S \cdot x_{R}+i \delta \cdot x_{R}} e^{2 S\left(x_{b}-x_{R}\right)} H_{m}^{(1)}\left(x_{R}\right) \\
-\frac{(S-u+i \delta)}{v}(S-i(\delta+1)) e^{S \cdot x_{R}-i \delta \cdot x_{R}}\left[1-e^{2 S\left(x_{b}-x_{R}\right)}\right] H_{m}^{(2)}\left(x_{R}\right)-2 S \frac{(S-u+i \delta)}{v} e^{S \cdot x_{R}-i \delta \cdot x_{R}} e^{2 S\left(x_{b}-x_{R}\right)} H_{m}^{(2)}\left(x_{R}\right)
\end{array}\right\}}
$$

\section{UNIFIED THEORY FOR CHIRPED CIRCULAR GRATING LASERS}

\subsection{Reflectivities of chirped circular Bragg gratings}

Eqs. (1) can also be solved in a more convenient way with the introduction of $\tilde{A}(x)=A(x) e^{-i \delta x}$ and $\tilde{B}(x)=B(x) e^{i \delta x}$ :

$$
\left\{\begin{array}{l}
\frac{d \tilde{A}(x)}{d x}=(u-i \delta) \tilde{A}(x)-v \tilde{B}(x) \\
\frac{d \tilde{B}(x)}{d x}=-(u-i \delta) \tilde{B}(x)+v \tilde{A}(x)
\end{array}\right.
$$

the generic solutions of which are

$$
\left\{\begin{array}{l}
\tilde{A}(x)=\tilde{A}(0) \frac{\sinh [S(x-L)]+\mathbb{C} \cosh [S(x-L)]}{-\sinh [S L]+\mathbb{C} \cosh [S L]} \\
\tilde{B}(x)=\frac{\tilde{A}(0)}{v} \cdot \frac{[(u-i \delta)-\mathbb{C} S] \sinh [S(x-L)]+[\mathbb{C}(u-i \delta)-S] \cosh [S(x-L)]}{-\sinh [S L]+\mathbb{C} \cosh [S L]},
\end{array}\right.
$$

where $S$ is defined previously as $S=\sqrt{(u-i \delta)^{2}-v^{2}}, \mathbb{C}$ is a constant to be determined by specific boundary conditions, and $L$ is a normalized length parameter.

The determination of the constant $\mathbb{C}$ in (11) requires the specific boundary conditions be applied. We investigate two typical boundary conditions to obtain $\mathbb{C}$ and the corresponding field reflectivity in each case. 


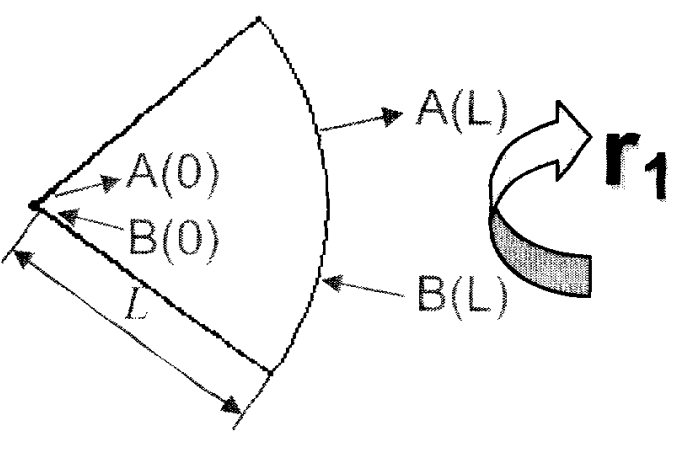

(a)

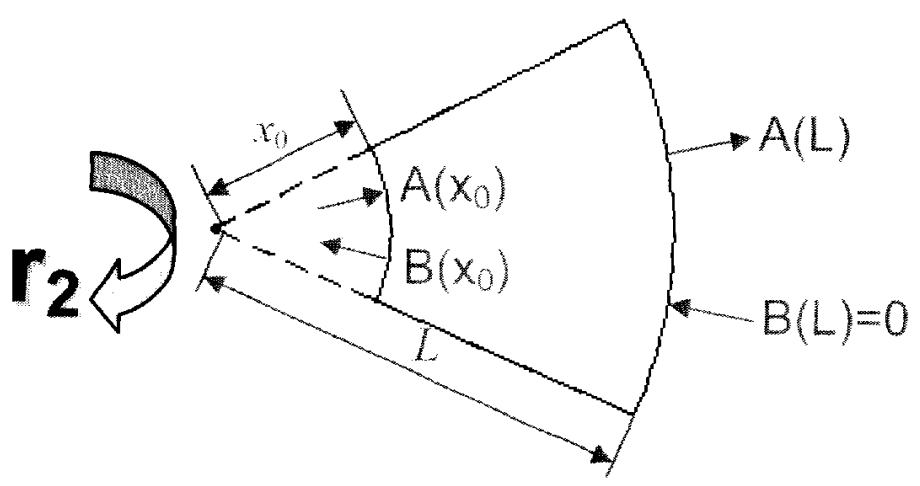

(b)

Fig. 2. Two types of boundary conditions for calculating reflectivities. (a) $A(0)=B(0), r_{1}(L)=A(L) / B(L) ;(\mathrm{b}) B(L)=0$, $r_{2}\left(x_{0}, L\right)=B\left(x_{0}\right) / A\left(x_{0}\right)$.

Case 1: As shown in Fig. 2(a), the grating extends from the center $x=0$ to $x=L$. An inward propagating wave with amplitude $B(L)$ impinges from outside on the grating. The reflectivity is defined as $r_{1}(L)=A(L) / B(L)$. In this case we have to ensure at the center $A(0)=B(0), \tilde{A}(0)=\tilde{B}(0)$, leading to $\mathbb{C}=\frac{(u-v-i \delta) \sinh [S L]+S \cosh [S L]}{S \sinh [S L]+(u-v-i \delta) \cosh [S L]}$ and to the reflectivity

$$
r_{1}(L)=\frac{A(L)}{B(L)}=\frac{\tilde{A}(L) e^{i \delta L}}{\tilde{B}(L) e^{-i \delta L}}=e^{2 i \delta L} \frac{(u-v-i \delta) \sinh [S L]+S \cosh [S L]}{-(u-v-i \delta) \sinh [S L]+S \cosh [S L]} .
$$

Case 2: As shown in Fig. 2(b), the grating extends from $x=x_{0}$ to $x=L$. An outward propagating wave with amplitude $A\left(x_{0}\right)$ impinges from inside on the grating. The reflectivity is defined as $r_{2}\left(x_{0}, L\right)=B\left(x_{0}\right) / A\left(x_{0}\right)$. Since there is no inward propagating wave coming from outside, we invoke $B(L)=0, \tilde{B}(L)=0$, leading to $\mathbb{C}=\frac{S}{u-i \delta}$ and to the reflectivity

$$
r_{2}\left(x_{0}, L\right)=\frac{B\left(x_{0}\right)}{A\left(x_{0}\right)}=\frac{\tilde{B}\left(x_{0}\right) e^{-i \delta x_{0}}}{\tilde{A}\left(x_{0}\right) e^{i \delta x_{0}}}=e^{-2 i \delta x_{0}} \frac{v \sinh \left[S\left(L-x_{0}\right)\right]}{(u-i \delta) \sinh \left[S\left(L-x_{0}\right)\right]-S \cosh \left[S\left(L-x_{0}\right)\right]} .
$$

It should be noted that, as seen from their definitions, the above reflectivities include the propagation phase.

\subsection{Reflectivity spectra of chirped circular Bragg gratings}

Active Bragg gratings with the inclusion of net gain can have the reflectivities (12) and (13) exceeding unity. As a case study, we take the coupling coefficients $h_{1}=0.0072+0.0108 i$ and $h_{2}=0.0601$ of a quarter-duty-cycle Hankel-phased circular grating, which was shown to be able to provide both large feedback for in-plane waves and a considerable mount of vertical radiation [7]. The two reflectivities (12) and (13) are analyzed for the case where the normalized grating length $(\beta \Delta \rho)$ is 100. Their amplitudes $\left|r_{1}(100)\right|$ and $\left|r_{2}\left(x_{0}, x_{0}+100\right)\right|$ are plotted in Fig. 3 with different gain $\left(g_{A}\right)$ levels. 

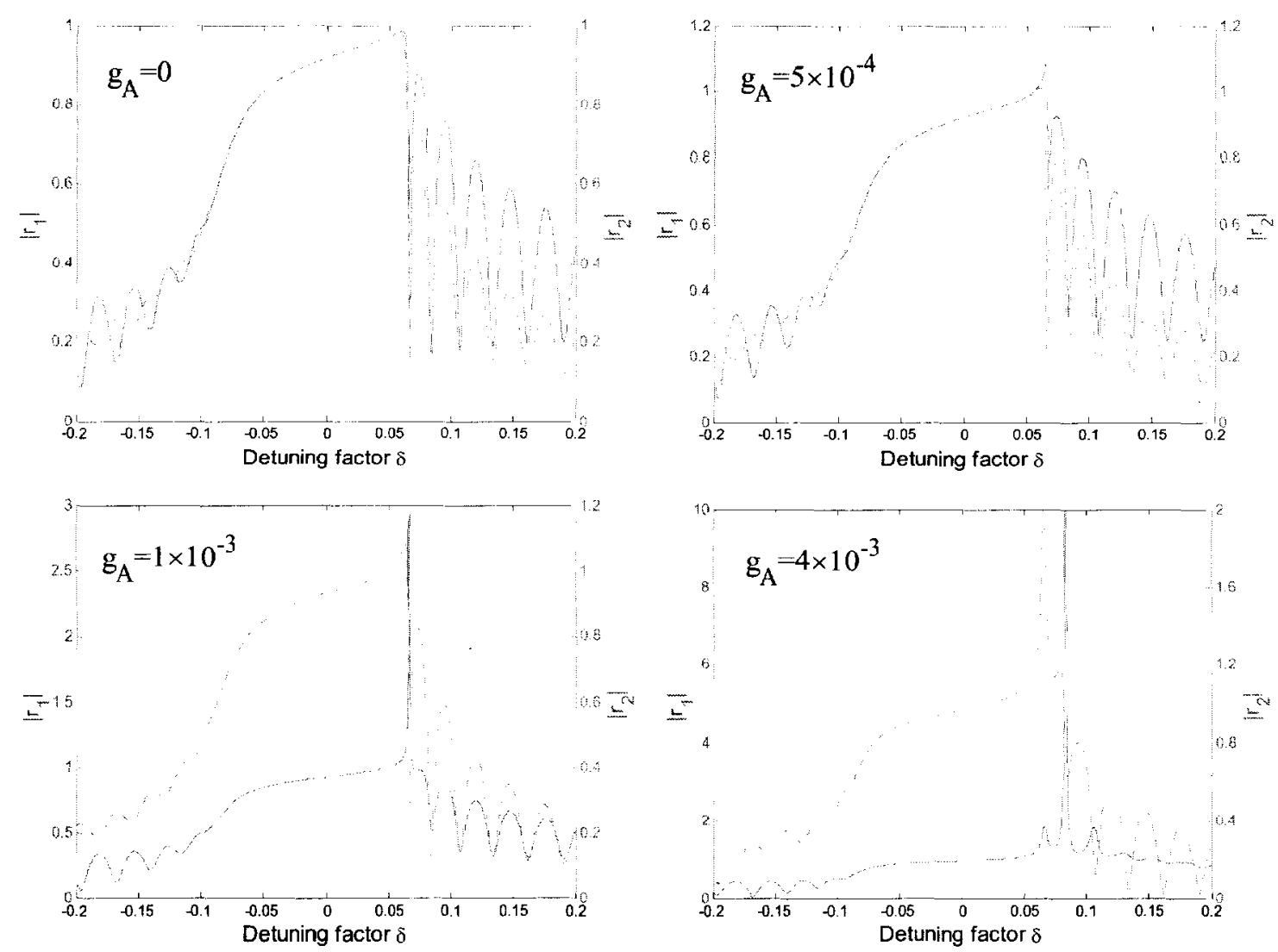

Fig. 3. Reflectivities $\left|r_{1}(100)\right|$ and $\left|r_{2}\left(x_{0} x_{0}+100\right)\right|$ for different gain levels $g_{A}=0,5 \times 10^{-4}, 1 \times 10^{-3}$, and $4 \times 10^{-3}$, in the presence of vertical radiation $\left(h_{1}=0.0072+0.0108 i\right.$ and $\left.h_{2}=0.0601\right)$.

In the passive case $\left(g_{A}=0\right)$, there exists a high reflection region at the center $(-0.06<\delta<0.06)$, corresponding to the band gap of the Bragg gratings. As the gain $\left(g_{A}\right)$ increases, both $\left|r_{1}\right|$ and $\left|r_{2}\right|$ grow and exceed unity first at one bandgap edge. Further increases in gain result in the existence of more lobes with peak value greater than unity. Additionally, both $\left|r_{1}\right|$ and $\left|r_{2}\right|$ are asymmetric with respect to $\delta=0$. The fringes with positive $\delta$ have more pronounced oscillations than those with negative $\delta$. This asymmetry in reflectivity spectra can be utilized for mode selection in surface-emitting circular grating lasers.

Similar to the case of a linear second-order Bragg grating, this mode selection is attributed to the involvement of vertical radiation coupling: the radiation fields coupled out from the in-plane outward- and inward- propagating cylindrical waves interfere constructively at one bandgap edge and destructively at the other. The difference in the interference behaviors of the radiation fields at the two bandgap edges is the mode selection mechanism [9].

To support the above statement, we also calculate and plot in Fig. 4 the reflectivities in the absence of radiation coupling $\left(h_{1}=0\right)$ for different gain levels. As can be seen, both $\left|r_{1}\right|$ and $\left|r_{2}\right|$ are symmetrically distributed with respect to $\delta=0$. Therefore it is the vertical radiation that makes the reflectivity spectra asymmetric and helps mode selection in the grating coupled surface emitting lasers. It has also been shown that engineering of this modal selection method is key to designing high-efficiency single-mode ring Bragg resonator lasers [8]. 

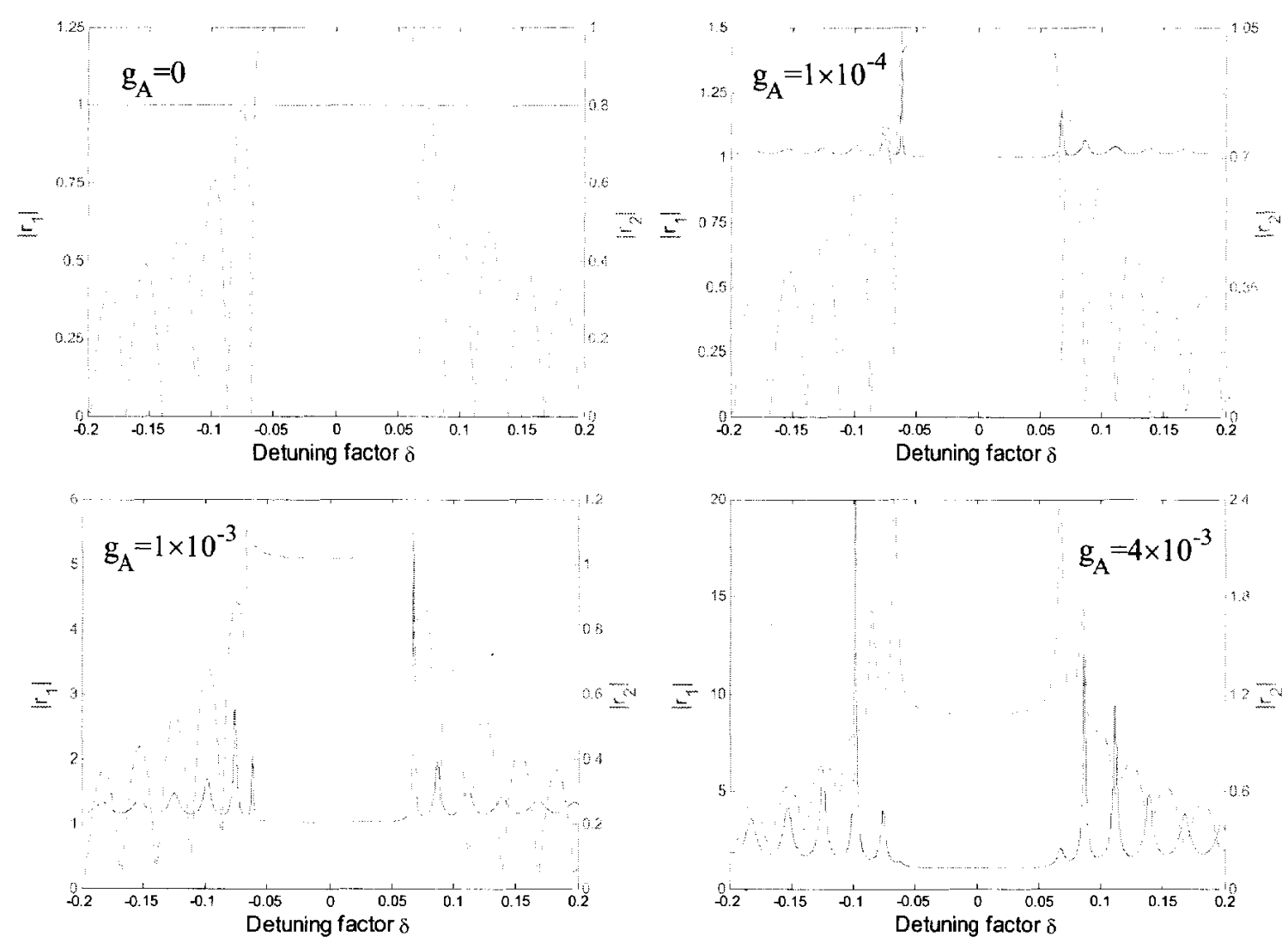

Fig. 4. Reflectivities $\left|r_{1}(100)\right|$ and $\left|r_{2}\left(x_{0}, x_{0}+100\right)\right|$ for different gain levels $g_{A}=0,1 \times 10^{-4}, 1 \times 10^{-3}$, and $4 \times 10^{-3}$, in the absence of vertical radiation $\left(h_{1}=0\right.$ and $\left.h_{2}=0.0601\right)$.

\subsection{Resonance conditions of circular DFB, disk-, and ring- Bragg resonator lasers}

With the reflectivities for different boundary conditions, it is easy to derive the resonance conditions for each circular grating laser configuration.

1. Circular DFB lasers:

The limiting cases $r_{1}\left(x_{b}\right) \rightarrow \infty$ or $r_{2}\left(0, x_{b}\right)=1$ lead to the same result

$$
\tanh \left[S x_{b}\right]=\frac{S}{u-v-i \delta}
$$

2. Disk Bragg resonator lasers:

Considering the radially propagating waves in the disk and taking the unity reflectivity at the center, the resonance condition is $1 \cdot e^{2 g_{A} x_{0}} \cdot r_{2}\left(x_{0}, x_{b}\right)=1$, which reads

$$
e^{2\left(g_{A}-i \hat{i}\right) x_{0}} \frac{v \sinh \left[S\left(x_{b}-x_{0}\right)\right]}{(u-i \delta) \sinh \left[S\left(x_{b}-x_{0}\right)\right]-S \cosh \left[S\left(x_{b}-x_{0}\right)\right]}=1 .
$$

3. Ring Bragg resonator lasers:

Considering the radially propagating waves in the annular defect, the resonance condition is $r_{1}\left(x_{L}\right) \cdot e^{2 g_{A}\left(x_{R}-x_{L}\right)} \cdot r_{2}\left(x_{R}, x_{b}\right)=1$, which reads

$$
e^{2\left(g_{A}-i \delta\right)\left(x_{R}-x_{L}\right)} \frac{(u-v-i \delta) \sinh \left[S x_{L}\right]+S \cosh \left[S x_{L}\right]}{-(u-v-i \delta) \sinh \left[S x_{L}\right]+S \cosh \left[S x_{L}\right]} \cdot \frac{v \sinh \left[S\left(x_{b}-x_{R}\right)\right]}{(u-i \delta) \sinh \left[S\left(x_{b}-x_{R}\right)\right]-S \cosh \left[S\left(x_{b}-x_{R}\right)\right]}=1 .
$$


The above resonance conditions govern the modes of the lasers of each type, and will be used to obtain the threshold gains $\left(g_{A}\right)$ and corresponding detuning factors $(\delta)$ of the laser modes. Though in much simpler forms, these resonance conditions (14)-(16) are essentially the same as the characteristic equations (5), (7), and (9). The argument follows: The foundation for validity of the "resonance condition theory" is the continuity of the amplitudes of the cylindrical waves at the interfaces. The in-plane electric field is expressed as $E(x)=A(x) H_{m}^{(1)}(x)+B(x) H_{m}^{(2)}(x)$. Its first derivative is given by $E^{\prime}(x)=\left[A^{\prime}(x)+i A(x)\right] H_{m}^{(1)}(x)+\left[B^{\prime}(x)-i B(x)\right] H_{m}^{(2)}(x)$. The continuity conditions for the outward- and inwardpropagating waves require that $A\left(x_{0}^{-}\right)=A\left(x_{0}^{+}\right)$and $B\left(x_{0}^{-}\right)=B\left(x_{0}^{+}\right)$hold at any interface $x_{0}$. These two independent restrictions adequately guarantee that $E\left(x_{0}^{-}\right)=E\left(x_{0}^{+}\right)$and $E^{\prime}\left(x_{0}^{-}\right)=E^{\prime}\left(x_{0}^{+}\right)$, which are the only requirements for matching the fields at the interfaces in deriving the characteristic equations $[7,8]$. The remaining two restrictions at the center $[A(0)-B(0)]$ and at the exterior boundary $\left[B\left(x_{b}\right)=0\right]$ are automatically satisfied by choosing proper reflectivities $\left(r_{1}\right.$ or $r_{2}$ ). Therefore, using the resonance condition theory, we can simplify and unify the modal analyses for the family of circular grating coupled surface emitting lasers.

\subsection{Modal field patterns, threshold gains, and frequency detuning factors}

Table 1. Modal field patterns, along with their threshold gains $\left(g_{A}\right)$ and frequency detuning factors $(\delta)$, of the circular DFB, disk-, and ring- Bragg resonator lasers for devices with $x_{b}=200$.

\begin{tabular}{|c|c|c|c|c|c|c|}
\hline \multicolumn{2}{|l|}{ Mode number } & 1 & 2 & 3 & 4 & 5 \\
\hline \multirow{3}{*}{$\begin{array}{l}\text { Circular DFB } \\
\text { laser }\end{array}$} & Modal field & & HIn & & 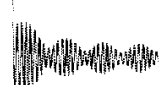 & m \\
\hline & $g_{A}\left(10^{-3}\right)$ & 0.283 & 1.03 & 2.04 & 3.11 & 4.12 \\
\hline & $\delta\left(10^{-3}\right)$ & 61.8 & 66.6 & 74.1 & 83.6 & 94.6 \\
\hline \multirow{3}{*}{$\begin{array}{l}\text { Disk Bragg } \\
\text { resonator laser }\end{array}$} & Modal field & & Whatrawam & mann & 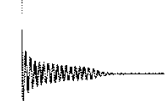 & 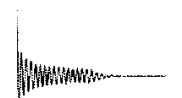 \\
\hline & $g_{A}\left(10^{-3}\right)$ & 0.127 & 0.288 & 0.454 & 0.690 & 1.21 \\
\hline & $\delta\left(10^{-3}\right)$ & 49.8 & 21.2 & -8.09 & -37.4 & -66.5 \\
\hline \multirow{3}{*}{$\begin{array}{l}\text { Ring Bragg } \\
\text { resonator laser }\end{array}$} & Modal field & & & & 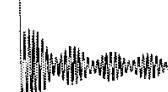 & 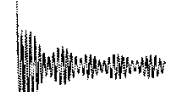 \\
\hline & $g_{A}\left(10^{-3}\right)$ & 0.457 & 1.06 & 1.92 & 3.14 & 4.09 \\
\hline & $\delta\left(10^{-3}\right)$ & 55.9 & 66.9 & 71.0 & 84.4 & 91.6 \\
\hline
\end{tabular}

Shown in Table 1 are the modal field patterns, together with the corresponding threshold gain values $\left(g_{A}\right)$ and frequency detuning factors $(\delta)$, of the circular DFB, disk-, and ring- Bragg resonator lasers numerically calculated from (14), (15), and (16). A typical device size of $x_{b}=200$ (corresponding to $\rho_{b}=17.5 \mu \mathrm{m}$ ) is assumed for all types of lasers. For the disk Bragg resonator laser, the inner disk radius $x_{0}$ is assumed to be $x_{b} / 2=100$. For the ring Bragg resonator laser, the annular defect is assumed to be located at the middle $x_{b} / 2=100$, and the defect width is set to be a wavelength of the cylindrical waves therein, yielding $x_{L}+x_{R}=x_{b}=200$ and $x_{R}-x_{L}=2 \pi$. The coupling coefficients are still taken to be $h_{1}=0.0072+0.0108 i$ and $h_{2}=0.0601$ as used in plotting the reflectivity spectra in Sec. 3.2. 
After comparison, we summarize the features of the modal properties of the three laser structures:

1. All the displayed modes of the circular DFB laser are in-band modes on one side of the band gap (all $\delta>0$ ). This is due to the aforementioned radiation-coupling-induced mode selection mechanism. Increased gain results in the excitation of higher-order modes.

2. All the displayed modes of the disk Bragg resonator laser are confined to the center disk with negligible peripheral power leakage and thus possess very low thresholds and very small modal volumes.

3. The modes of the ring Bragg resonator laser, with the exception of the fundamental defect mode, all resemble their counterparts of the circular DFB laser. The defect mode has a larger threshold gain than the fundamental mode of the circular DFB device, but it possesses a much higher emission efficiency due to its unique modal profile.

\section{CONCLUSIONS}

A unified theory for solving and comparing modal properties of surface-emitting chirped circular grating lasers was developed and presented. Two types of reflectivities were obtained from the coupled-wave equations for chirped circular Bragg gratings under two typical boundary conditions. The asymmetry in the reflectivity spectra shows evidence of modal selection due to the involvement of vertical radiation. The resonance conditions were then derived for three types of circular grating lasers: circular DFB, disk-, and ring- Bragg resonator lasers. Though in simpler formats, those resonance conditions are essentially the same as the characteristic equations derived in previous works. With the governing resonance conditions, the modal properties, including the modal field patterns, threshold gains, and detuning factors, of the three types of circular grating lasers were obtained and compared.

\section{REFERENCES}

[1] R. Coccioli, M. Boroditsky, K. W. Kim, Y. Rahmat-Samii, and E. Yablonovitch, "Smallest possible electromagnetic mode volume in a dielectric cavity," IEE Proc.-Optoelectron. 145, 391-397 (1998).

[2] E. A. J. Marcatili, "Bends in Optical Dielectric Guides," Bell Syst. Tech. J. 48, 2103-2132 (1969).

[3] J. Scheuer and A. Yariv, "Coupled-Waves Approach to the Design and Analysis of Bragg and Photonic Crystal Annular Resonators," IEEE J. Quantum Electron. 39, 1555-1562 (2003).

[4] J. Scheuer and A. Yariv, "Annular Bragg defect mode resonators," J. Opt. Soc. Am. B 20, 2285-2291 (2003).

[5] J. Scheuer, W. M. J. Green, G. DeRose, and A. Yariv, "Low-threshold two-dimensional annular Bragg lasers," Opt. Lett. 29, 2641-2643 (2004).

[6] J. Scheuer, W. M. J. Green, G. A. DeRose, and A. Yariv, "Lasing from a circular Bragg nanocavity with an ultrasmall modal volume," Appl. Phys. Lett. 86, 251101 (2005).

[7] X. K. Sun, J. Scheuer, and A. Yariv, "Optimal design and reduced threshold in vertically emitting circular Bragg disk resonator lasers," IEEE J. Sel. Top. Quantum Electron. 13, 359-366 (2007).

[8] X. K. Sun and A. Yariv, "Modal properties and modal control in vertically emitting annular Bragg lasers," Opt. Express 15, 17323-17333 (2007).

[9] R. F. Kazarinov and C. H. Henry, "Second-Order Distributed Feedback Lasers with Mode Selection Provided by First-Order Radiation Losses," IEEE J. Quantum Electron. 21, 144-150 (1985). 